\title{
The clinical management of hyponatraemia
}

\author{
David M Williams, ${ }^{1}$ Maire Gallagher, ${ }^{1}$ Joel Handley, ${ }^{1}$ Jeffrey W Stephens ${ }^{1,2}$
}

${ }^{1}$ Department of Diabetes \& Endocrinology, Morriston Hospital, ABMU Health Board, Swansea, UK

${ }^{2}$ Department of Medicine, Welsh Institute of Metabolic and Obesity Surgery, Swansea, UK

\section{Correspondence to} Dr David M Williams, Department of Diabetes \& Endocrinology, Morriston Hospital, Swansea SA6 6NL, UK; david.williams@doctors. org.uk

Received 14 October 2015 Revised 28 January 2016 Accepted 11 March 2016 Published Online First 4 April 2016

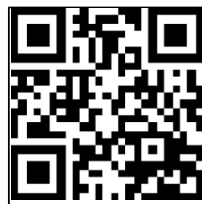

Editor's choice Scan to access mo free content
To cite: Williams DM, Gallagher M, Handley J, et al. Postgrad Med J 2016;92:407-411.

\begin{abstract}
Hyponatraemia is the most common electrolyte disorder seen in clinical practice and the consequences can range from minor symptoms to life-threatening complications including seizures and cardiorespiratory distress. These effects occur as a result of fluid shifts due to deranged serum tonicity and subsequent cerebral oedema. The appropriate assessment and management of patients with hyponatraemia is not always achieved in clinical practice, which is partly related to challenges in teaching with limited clinical guidance. Recently, the European Society of Endocrinology, European Society of Intensive Care Medicine and European Renal AssociationEuropean Dialysis and Transplant Association produced clinical practice guidelines to focus on appropriate investigation and management of these patients. Within this manuscript, we highlight the key points from these guidelines, which are most pertinent to doctors of all specialties to improve the care of patients with this common electrolyte disorder.
\end{abstract}

\section{BACKGROUND}

Hyponatraemia is the most common electrolyte disorder seen in hospital inpatients in the UK. Approximately $15 \%$ of inpatients have a serum sodium concentration $\quad<135 \mathrm{mmol} / \mathrm{L}, \quad 4 \%$ $<130 \mathrm{mmol} / \mathrm{L}$ and $2 \%<125 \mathrm{mmol} / \mathrm{L}^{1}$ This prevalence is greater in the acute critical setting such as intensive care, where approximately $30 \%$ of patients have a serum sodium $<134 \mathrm{mmol} / \mathrm{L}^{2}$ Clinical presentation and subsequent management are dependent on the nature and severity of the clinical symptoms and signs, and the aetiology of the hyponatraemia. Patients with acute, severe symptoms will require specialist input for rapid correction of the serum sodium. Such patients are also at a higher risk of morbidity, length of hospital stay and mortality. ${ }^{13}$ Patients with hyponatraemia are seven times more likely to die than those without hyponatraemia in hospital. ${ }^{4}$ Hospital doctors regularly manage patients with hyponatraemia across a range of inpatient wards, and therefore the management of this common electrolyte disorder is important in routine clinical practice. However, optimal management of these patients is not always achieved, which is in part due to a lack of knowledge. Within this manuscript, we summarise the key points from the joint guidelines from the European Society of Endocrinology, European Society of Intensive Care Medicine and European Renal AssociationEuropean Dialysis and Transplant Association. ${ }^{5}$

Hyponatraemia is defined as serum sodium $<135 \mathrm{mmol} / \mathrm{L}$. It may be classified temporally (acute $<48 \mathrm{~h}$; chronic $>48 \mathrm{~h}$ ) or by the absolute serum sodium level. ${ }^{5}$ Mild (130-135 mmol/L), moderate $(125-130 \mathrm{mmol} / \mathrm{L})$ or severe $(<125 \mathrm{mmol} / \mathrm{L})$ hyponatraemia presents with non-specific signs and symptoms, and are summarised in table 1. However, patients should be assessed and treated on the basis of their symptoms rather than the absolute serum sodium level or time frame in which hyponatraemia develops.

\section{CLINICAL FEATURES}

The clinical presentation of hyponatraemia is often non-specific but may include life-threatening seizures and cardiorespiratory arrest. Patients with mild-to-moderate hyponatraemia may have symptoms such as nausea, confusion and headache, whereas those with moderate to severe hyponatraemia will present with vomiting, cardiorespiratory distress, seizures and reduced consciousness, ${ }^{3}{ }^{5}$ as summarised in table 1. A patient is likely to present with more severe symptoms if they have acuteonset hyponatraemia as cerebral oedema develops secondary to the reduced serum osmolality. In chronic hyponatraemia there is lower risk of neurological dysfunction as the brain initiates counterregulatory mechanisms to reduce cerebral oedema, typically over a $24-48 \mathrm{~h}$ period. ${ }^{3}$ This explains the rationale for classifying acute hyponatraemia as that which occurs within $48 \mathrm{~h}$ and the relative severity of the symptoms that patients' develop. ${ }^{5}$ Importantly, there must be a normal serum sodium concentration demonstrated in the previous $48 \mathrm{~h}$ for acute hyponatraemia to be diagnosed, otherwise the assumption must be that the patient has a chronic hyponatraemia.

\section{THE AETIOLOGY AND ASSESSMENT OF HYPONATRAEMIA}

A detailed clinical assessment of patients with mild-to-moderate hyponatraemia is essential, as aetiology will guide clinical management. Patients with severe or life-threatening hyponatraemia may require rapid correction of their serum sodium (see management of patients with severe symptoms section), and therefore specialist input may be required prior to obtaining a clinical assessment.

Hyponatraemia in the presence of high serum osmolality may occur as a consequence of hyperglycaemia, termed hypertonic hyponatraemia. Hyperglycaemia may result in a fluid shift by osmosis from the intracellular to the extracellular fluid (ECF) compartment, thus diluting serum sodium levels. ${ }^{6}$ When hyperglycaemia is corrected, the sodium concentration will correct as fluid returns by osmosis to the intracellular compartment. Therefore, when treating patients with hyperglycaemia it is important to control the rate at which plasma glucose is lowered, to minimise the associated risk of cerebral oedema that can occur. ${ }^{7}$

Once non-hypotonic hyponatraemia has been excluded, it is important to measure the serum osmolality and both the urine osmolality and 
Table 1 Summary of clinical features associated with hyponatraemia

\begin{tabular}{lll}
\hline Subtle symptoms & $\begin{array}{l}\text { Mild } \\
\text { symptoms }\end{array}$ & Severe symptoms \\
\hline Gait abnormalities & Nausea & Vomiting \\
Falls & Confusion & $>$ Cardiorespiratory distress \\
Reduced concentration & Headache & Abnormal and deep sleep \\
Cognitive deficits & & \\
Increased osteoporosis and & & \\
fractures & & \\
\hline
\end{tabular}

sodium concentration. In the instance the serum osmolality is $>275 \mathrm{mOsmol} / \mathrm{kg}$ then isotonic or hypertonic hyponatraemia is present. Conversely, if the serum osmolality is $<275 \mathrm{mOsmol} /$ $\mathrm{kg}$, the patient has a hypotonic hyponatraemia. The urine osmolality and urinary sodium can help identify the aetiology. In most instances when the urine osmolality $<100 \mathrm{mOsmol} / \mathrm{kg}$, it is likely that the patient has excess water intake. Commonly, when the urine osmolality $>100 \mathrm{mOsmol} / \mathrm{kg}$ and urinary sodium is $<30 \mathrm{mmol} / \mathrm{L}$, there is a low effective arterial volume. In those patients with a urine osmolality $>100 \mathrm{mOsmol} / \mathrm{kg}$ and a urinary sodium $>30 \mathrm{mmol} / \mathrm{L}$, assessment of the patient's ECF status should be undertaken (see assessing ECF status section). If the ECF volume is low, the patient's hyponatraemia may be secondary to diuretic use, primary adrenal insufficiency, vomiting and cerebral or renal salt wasting. For patients who are clinically euvolaemic, consider the syndrome of inappropriate diuresis (SIAD), secondary adrenal insufficiency, hypothyroidism as well as drug-induced aetiologies. This is shown in figure 1 .

\section{ASSESSING ECF STATUS}

The assessment of ECF status is often difficult, and is most useful in patients with hyponatraemia when urine osmolality and urinary sodium levels can also be reviewed. The aetiological basis of hyponatraemia can be classified by the fluid status of the patient as hypovolaemic, euvolaemic or hypervolaemic hyponatraemia. Major causes of hypovolaemic hyponatraemia include diuretics, renal failure and adrenal insufficiency; causes of euvolaemic hyponatraemia include SIAD and hypothyroidism; hypervolaemic hyponatraemia is caused by chronic medical conditions such as cardiac failure, hepatic disease and renal disease. ${ }^{8}$ Table 2 summarises the causes of hyponatraemia by fluid status.

The clinical history will guide the assessment as the patient may have had diarrhoea, vomiting, polyuria or a pre-existing medical condition such as cardiac or renal failure. At this point in the assessment, ask about symptoms of hyponatraemia as this may change the approach to management (figure 2). Signs of hypovolaemia include dry mucous membranes, tachycardia and hypotension. Patients with hypervolaemia present with raised jugular venous pressure, peripheral and pulmonary oedema. ${ }^{9}$ Euvolaemia is determined primarily through the absence of other signs. However, clinical examination alone to assess a patient's fluid status is often difficult, especially when assessing elderly patients who may demonstrate inconsistent signs. ${ }^{10}$ Therefore, consideration of body weight, fluid balance charts and intravenous fluid prescription is also essential.

In many cases of hyponatraemia there is an increased secretion of antidiuretic hormone (ADH), which can further reduce the serum sodium level, termed SIAD. In those with hypovolaemic hyponatraemia, $\mathrm{ADH}$ is released from the posterior pituitary gland via a baroceptor-mediated reflex, which increases renal water reabsorption. This will help to restore intravascular volume, but at the cost of reducing serum sodium levels and the serum osmolality further. In patients with hypervolaemia and hyponatraemia there is often a concurrent diagnosis of cardiac failure or hepatic impairment, resulting in reduced cardiac output and peripheral vasodilation, respectively. This

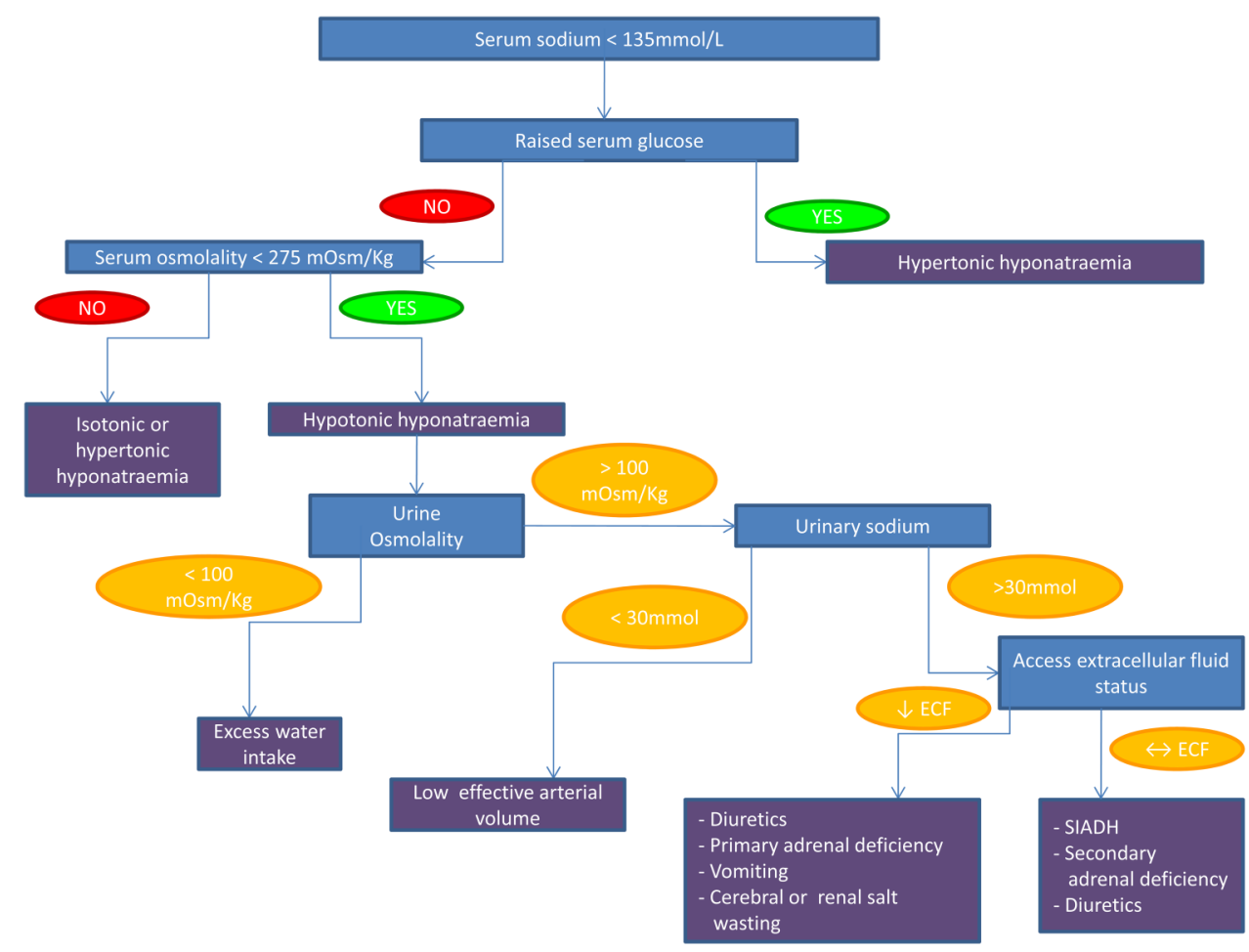

Figure 1 How to investigate hyponatraemia. ECF, extracellular fluid. 
Table 2 Classification of hyponatraemia based on extracellular fluid volume

\begin{tabular}{lll}
\hline Hypovolaemia & Euvolaemia & Hypervolaemia \\
\hline $\begin{array}{l}\text { Gastrointestinal loss } \\
\text { (vomiting, diarrhoea) }\end{array}$ & $\begin{array}{l}\text { Syndrome of } \\
\text { inappropriate diuresis } \\
\text { Diuretics (thiazides) }\end{array}$ & Renal failure \\
Primary adrenal insufficiency & Seart failure \\
Cerebral salt wasting & insufficiency adrenal & Hypothyroidism \\
Renal disease & High water/low solute & \\
$\begin{array}{l}\text { (salt-losing nephropathies) } \\
\text { 'Third spacing' } \\
\text { (pancreatitis, sepsis, bowel } \\
\text { obstruction) }\end{array}$ & intake(primary \\
polydipsia) & \\
\hline
\end{tabular}

causes a similar reflex as described above and further water reabsorption resulting in both fluid overload and a chronic hyponatraemia. ${ }^{11}$

In practice, it is difficult to know the prevalence of the different causes of hyponatraemia because often a full clinical assessment has not been completed and the causes differ between specialties and patient subgroups. Commoner causes include medication-induced hyponatraemia ${ }^{8}$ and SIAD. A recent study in a sample of elderly patients admitted with fragility fractures, observed that hyponatraemia in $>75 \%$ was potentially related to the use of thiazide diuretics. ${ }^{12}$ Other medications that may result in hyponatraemia include antipsychotics, antiepileptic medications, selective serotonin reuptake inhibitors, ACE inhibitors, angiotensin receptor blockers and proton-pump inhibitors. SIAD is described in further detail below.

Therefore, patients with hyponatraemia should have serum urea and electrolytes, glucose, osmolality and thyroid function tests measured in addition to urine analysis for osmolality and sodium concentrations. In patients with suspected adrenal insufficiency, one should also consider testing a 09:00 h serum cortisol level or performing a short synacthen test.

\section{SYNDROME OF INAPPROPRIATE DIURESIS}

This syndrome results from the excessive, unregulated secretion of $\mathrm{ADH}$, which results in the kidney's inability to effectively dilute urine. Reduced serum osmolality and hyponatraemia result from the excess water reabsorption in the renal tubules. SIAD is the most common cause of euvolaemic hyponatraemia, ${ }^{13}$ but should be considered as a diagnosis of exclusion after other causes of hyponatraemia have been excluded. ${ }^{5}$ Beware that the other conditions discussed previously may result in the appropriate increased secretion of $\mathrm{ADH}$ and may therefore present with a similar clinical picture. ${ }^{14}$

The diagnosis of SIAD requires the exclusion of the other causes of hyponatraemia, which result in the physiological secretion of $\mathrm{ADH}$. The biochemical markers suggestive of increased $\mathrm{ADH}$ secretion include a serum osmolality $<275 \mathrm{mOsmol} / \mathrm{kg}$ with urine osmolality $>100 \mathrm{mOsmol} / \mathrm{kg}$. The urine osmolality is inappropriately concentrated relative to the plasma osmolality, and the urinary sodium concentration is typically $>30 \mathrm{mmol} / \mathrm{L}$. The patient should be clinically euvolaemic and have normal adrenal, thyroid and pituitary function. These patients should also have normal renal function and no recent history of diuretic use. $^{13} 15$

There are many causes of SIAD, which are also summarised in table 3. These include malignancies of the lung, gastrointestinal and genitourinary tracts; pneumonia; neurological disorders such meningitis/encephalitis, subdural haematomas and stroke. Medications such as antidepressants, antipsychotics and antiepileptic medications are also known to cause SIAD. ${ }^{16}$ However, in practice there is often no attributable cause.

\section{CEREBRAL/RENAL SALT-WASTING SYNDROME}

Cerebral/renal salt-wasting syndrome is a rare condition which leads to a mild-to-moderate hyponatraemia, and is commonly associated with intracranial bleeds (subarachnoid haemorrhage, subdural haematomas), cerebral injury or brain tumours. The term cerebral salt-wasting syndrome is commonly used due to its significant association with the cerebral pathologies listed.

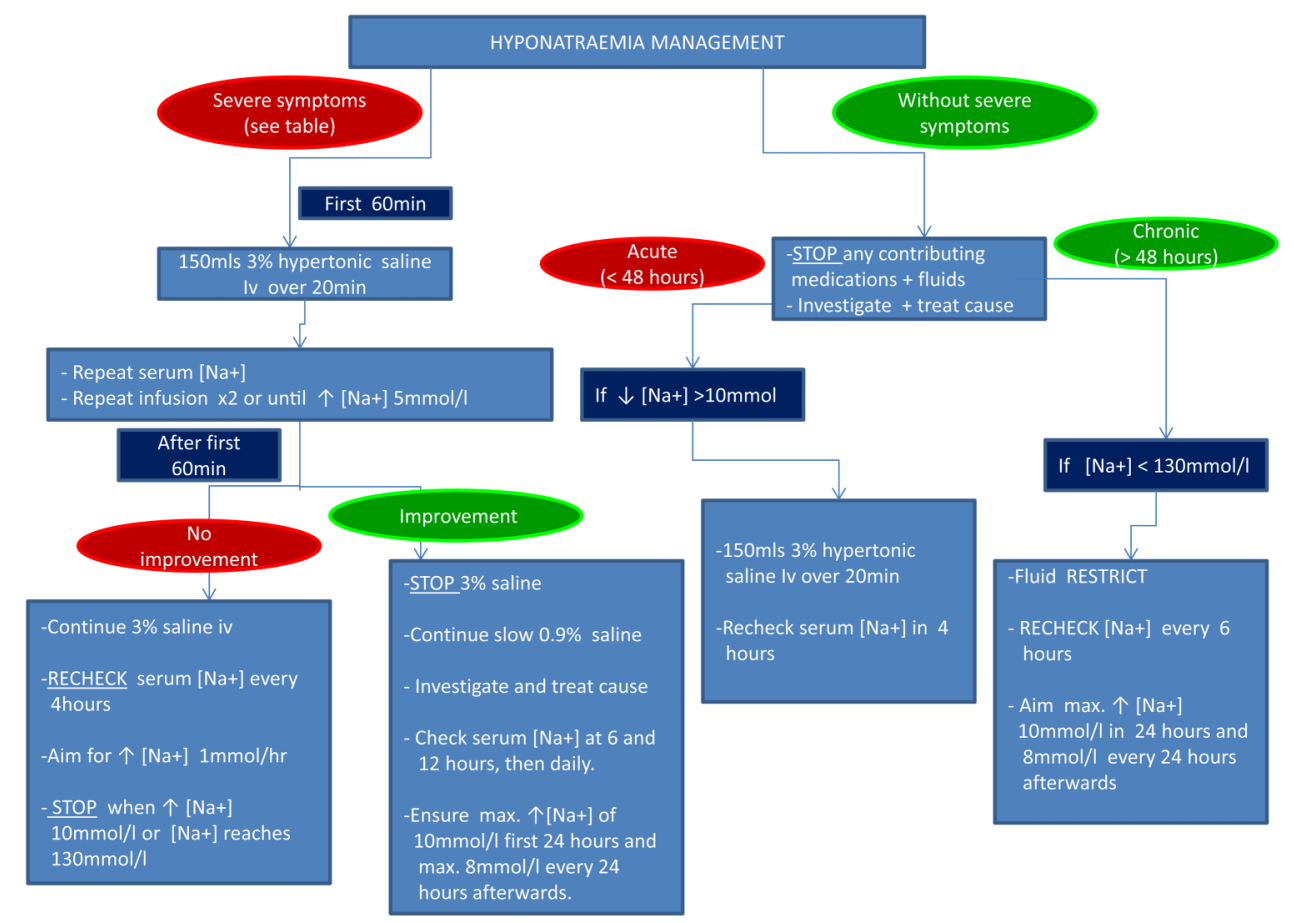

Figure 2 Management of hyponatraemia. 
Table 3 Summary of the aetiology of syndrome of inappropriate diuresis

\begin{tabular}{lll}
\hline Malignant disorders & Pulmonary disorders & Central nervous system disorders \\
\hline Carcinoma & Infections & Infection \\
Lung & Bacterial or viral pneumonia & Meningitis \\
Gastrointestinal tract & Tuberculosis & Esthma
\end{tabular}

However, the syndrome often occurs without cerebral pathology, and as such the term renal salt-wasting syndrome is often considered to be more appropriate. ${ }^{17}$ The mechanism of the syndrome is thought to be initiated by the release of a natriuretic factor which results in the increased renal excretion of sodium and subsequent volume depletion. As a result of the subsequent intravascular volume depletion, there is release of $\mathrm{ADH}$ and increased renin-angiotensin-aldosterone activity to reduce the rate of diuresis. ${ }^{18}$ Crucially, it must be recognised that the release of $\mathrm{ADH}$ in this setting is an appropriate response to the volume depletion, and this often makes it clinically difficult to differentiate cerebral/renal salt-wasting syndrome from SIAD.

Investigation of these patients will demonstrate similar laboratory findings as observed in patients with SIAD, low serum osmolality $(<275 \mathrm{mOsmol} / \mathrm{kg})$, high urine osmolality $(>100 \mathrm{mOsmol} / \mathrm{kg})$ and high urinary sodium concentration (>30 mmol/L). However, on examination, patients with cerebral salt wasting are dehydrated, and as described above, patients with SIAD are euvolaemic. Treatment of cerebral salt-wasting syndrome is usually best achieved with intravenous supplementation of $0.9 \%$ sodium chloride, and specialist use of fludrocortisone. ${ }^{19}$ In most patients there is spontaneous resolution within 2 weeks, though in elderly patients prolonged treatment may be required.

\section{APPROACHES TO MANAGEMENT}

The clinical management of patients with acute hyponatraemia takes account of the patient's symptoms, the duration of onset (ie, acute or chronic), fluid balance and absolute sodium level. The available guidelines consider the management of patients with severe and moderately severe symptoms separately as summarised in figure $2 .^{5}$

\section{MANAGEMENT OF PATIENTS WITH SEVERE SYMPTOMS}

The management of patients with hyponatraemia with severe symptoms is best achieved by senior and specialist doctors working in a closely monitored environment in which there is easily available blood monitoring, and as such doctors who are uncertain of appropriate management strategies should seek help from appropriate medical or critical care teams as early as possible. Treatment is initiated with an intravenous infusion of $150 \mathrm{~mL} \mathrm{3 \%}$ hypertonic sodium chloride over $20 \mathrm{~min}$. Following the infusion the serum sodium is measured and a further $150 \mathrm{~mL}$ infusion can be administered while waiting for the result. If the patient's symptoms have not adequately improved, continue with the intravenous hypertonic saline infusions, regularly checking the serum sodium at least every $4 \mathrm{~h}$. The hypertonic saline infusions should be stopped when the patient's symptoms improve, the serum sodium concentration increases by $10 \mathrm{mmol} / \mathrm{L}$ or the serum sodium is $130 \mathrm{mmol} / \mathrm{L}$. In patients who respond to treatment, consider a slow infusion of $0.9 \%$ sodium chloride and cause-specific treatment. In these patients, the serum sodium should be checked after $6 \mathrm{~h}$, and subsequently at $12 \mathrm{~h}$, and daily thereafter. If the patient requires concurrent fluid resuscitation, this over-rides the risk of rapid correction of hyponatraemia (section below).

\section{MANAGEMENT OF PATIENTS WITHOUT SEVERE SYMPTOMS}

The clinical management of hyponatraemia without severe symptoms is guided by the rate of onset. If the onset is acute, consider stopping any contributing fluids and medications. If the drop in serum sodium is $>10 \mathrm{mmol} / \mathrm{L}$ consider giving $150 \mathrm{~mL} \mathrm{3 \%}$ sodium chloride over $20 \mathrm{~min}$ intravenously and measure the serum sodium after $4 \mathrm{~h}$. Following this, undertake the investigations as described above and initiate cause-specific treatment. It should be noted that this approach in management should be undertaken only with specialist supervision and facilities to regularly check serum sodium levels.

In patients with chronic hyponatraemia without severe symptoms management should consist of stopping any contributing non-essential medications or fluids. Further treatment takes account of the fluid status. Patients with hypervolaemia or SIAD are best managed with fluid restriction. If there is no improvement, low-dose loop diuretics and oral sodium chloride should be started. In these patients always consider the causes of SIAD and treat appropriately. Patients with hypovolaemia should be given intravenous $0.9 \%$ sodium chloride or a balanced crystalloid solution at a rate of $0.5-1.0 \mathrm{~mL} / \mathrm{kg} / \mathrm{h}$. Importantly, asymptomatic patients with mild hyponatraemia do not warrant aggressive treatment. Guidelines frequently reference the patient's symptoms as the most important factor when considering treatment of hyponatraemia and treatment of mild hyponatraemia is only warranted when symptomatic.

\section{RAPID CORRECTION OF SERUM SODIUM}

The treatments outlined above may result in a rapid correction of the serum sodium and serum osmolality. In rare cases, this may precipitate osmotic demyelination syndrome (ODS), which presents in patients with changes in mental status, rapid quadriplegia and dysphagia. ${ }^{20}$ There is an increased risk of developing ODS in patients with SIAD, significant burns and those who chronically abuse alcohol. ${ }^{21}$ While patients with a rapidly corrected chronic hyponatraemia are considerably more likely to 
develop ODS, ${ }^{22}$ there is a small number of patients described in the literature who have developed ODS following rapid correction of an acute hyponatraemia. ${ }^{23}$ As such, we should bear in mind the risks of rapidly correcting serum sodium levels in all patients with hyponatraemia.

To minimise the risk, guidelines recommend stopping the treatment of hyponatraemia if there is an increase in serum sodium $>10 \mathrm{mmol} / \mathrm{L}$ in the first $24 \mathrm{~h}$ or $>8 \mathrm{mmol} / \mathrm{L}$ in each subsequent $24 \mathrm{~h}$. Close monitoring of the patient's serum electrolytes is therefore required, and as such aggressive management should only be sought in an appropriately close-monitored environment. In these circumstances, it is essential to seek expert advice on whether to begin an infusion of electrolyte-free solutions (eg, glucose solutions) while under strict fluid balance and urine output monitoring.

\section{CONCLUSION}

Hyponatraemia is a disorder which often presents nonspecifically and has a considerable aetiological basis. Appropriate management of hyponatraemia takes account of the patient's symptoms and the acute or chronic nature of onset. Appropriately educating doctors is important in improving clinical management, which is often suboptimal in practice and essential in minimising the associated morbidity and mortality of the condition. The new guidelines summarised here will hopefully reduce the previous difficulty in teaching this important clinical topic to both medical students and doctors, which will ultimately improve the treatment of this common electrolyte disorder.

\section{Main messages}

- Hyponatraemia is a complex metabolic disorder which carries significant morbidity and mortality in hospital inpatients.

- Patients with hyponatraemia require thorough examination and investigation to ascertain the cause, which guides further management appropriately.

- Management is guided by the absolute serum sodium level or underlying disorder, and by the patient's symptomatology.

\section{Key reference}

- Spasovski G, Vanholder R, Allolio B, et al. Clinical practice guideline on diagnosis and treatment of hyponatraemia. Eur J Endocrinol 2014;170:G1-47.
Contributors DMW planned, wrote and edited the manuscript. MG created the figures and tables used in the article, and critically edited the manuscript. JH planned and critically edited the manuscript. JWS supervised the writing of the article and critically edited the completed manuscript. All authors give approval of the version to be published.

Competing interests None declared.

Provenance and peer review Not commissioned; externally peer reviewed.

\section{REFERENCES}

1 Asadollahi K, Beeching N, Gill G. Hyponatraemia as a risk factor for hospital mortality. QJM 2006;99:877-80.

2 DeVita MV, Gardenswartz MH, Konecky A, et al. Incidence and etiology of hyponatraemia in an intensive care unit. Clin Nephrol 1990;34: $163-6$

3 Thompson CJ. Hyponatraemia: new associations and new treatments. Eur J Endocrinol 2010;162:S1-3.

4 Tierney WM, Martin DK, Greenlee MC, et al. The prognosis of hyponatremia at hospital admission. J Gen Intern Med 1986;1:380-5.

5 Spasovski G, Vanholder R, Allolio B, et al. Clinical practice guideline on diagnosis and treatment of hyponatraemia. Eur J Endocrinol 2014;170:G1-47.

6 Hillier TA, Abbott RD, Barrett EJ. Hyponatremia: evaluating the correction factor for hyperglycemia. Am J Med 1999;106:399-403.

7 Hoorn EJ, Carlotti AP, Costa LA, et al. Preventing a drop in effective plasma osmolality to minimize the likelihood of cerebral edema during treatment of children with diabetic ketoacidosis. J Pediatr 2007;150:467-73.

8 Freda BJ, Davidson MB, Hall PM. Evaluation of hyponatremia: a little physiology goes a long way. Cleve Clin J Med 2004;71:639-50.

9 Frank Peacock W, Soto KM. Current technique of fluid status assessment. Congest Heart Fail 2010;16(Suppl 1):S45-51.

10 Hoyle GE, Chua M, Soiza RL. Volaemic assessment of the elderly hyponatraemic patient: reliability of clinical assessment and validation of bioelectrical impedance analysis. QJM 2011;104:35-9.

11 Schrier RW. Body fluid volume regulation in health and disease: a unifying hypothesis. Ann Intern Med 1990;113:155-9.

12 Cumming K, Hoyle GE, Hutchison JD, et al. Prevalence, incidence and etiology of hyponatremia in elderly patients with fragility fractures. PLOS ONE 2014;9: e88272.

13 Schwartz WB, Bennett W, Curelop S, et al. A syndrome of renal sodium loss and hyponatremia probably resulting from inappropriate secretion of antidiuretic hormone. Am J Med 1957;23:529-42.

14 Biswas M, Davies JS. Hyponatraemia in clinical practice. Postgrad Med J 2007;83:373-8

15 Janicic N, Verbalis JG. Evaluation and management of hypoosmolality in hospitalized patients. Endocrinol Metab Clin North Am 2003;32:459-81.

16 Liamis $\mathrm{G}$, Milionis $\mathrm{H}$, Elisaf M. A review of drug-induced hyponatraemia. Am J Kidney Dis 2008;52:144-53.

17 Maesaka JK, Imbriano L, Ali NM, et al. Is it cerebral or renal salt wasting? Kidney Int 2009;76:934-8.

18 Maesaka JK, Imbriano L, Mattana J, et al. Differentiating SIADH from Cerebral/Renal Salt Wasting: Failure of the Volume Approach and Need for a New Approach to Hyponatremia. J Clin Med 2014;3:1373-85.

19 Betjes MG. Hyponatremia in acute brain disease: the cerebral salt wasting syndrome. Eur J Intern Med 2002;13:9-14.

20 King JD, Rosner MH. Osmotic demyelination syndrome. Am J Med Sci 2010;339:561-7.

21 Lampl C, Yazdi K. Central pontine myelinolysis. Eur Neurol 2002;47:3-10.

22 Singh TD, Fugate JE, Rabinstein AA. Central pontine and extrapontine myelinolysis: a systematic review. Eur I Neurol 2014;21:1443-50.

23 Martin RJ. Central pontine and extrapontine myelinolysis: the osmotic demyelination syndromes. J Neurol Neurosurg Psychiatr 2004;75(Suppl 3):iii22-8. 